\title{
The role of the miR-21-5p-mediated inflammatory pathway in ulcerative colitis
}

\author{
XIAOHONG LU, YUANJIE YU and SHIYUN TAN \\ Department of Gastroenterology, Renmin Hospital of Wuhan University, \\ Hubei Key Laboratory of Digestive System Disease, Wuhan, Hubei 430060, P.R. China
}

Received January 2, 2019; Accepted August 20, 2019

DOI: $10.3892 /$ etm.2019.8277

\begin{abstract}
Ulcerative colitis (UC), a major type of inflammatory bowel disease, is also a chronic non-specific intestinal inflammation condition of unknown etiology. The pathogenesis of UC is closely associated with immune abnormalities, inflammatory damage and genetics. The present study aimed to explore the effects of microRNA (miR)-21-5p on the interleukin-6 (IL-6) receptor (IL6R)/signal transducer and activator of transcription (STAT3) signal pathway in UC, in order to identify a highly effective treatment for UC. A total of 45 patients with UC and 45 healthy controls were recruited for the present study. The expression levels of miR-21-5p and STAT3 in the sera of patients with UC and healthy controls were determined by reverse transcription-quantitative polymerase chain reaction (RT-qPCR). A UC rat model was established using dextran sulfate sodium. Following lipopolysaccharide (LPS) treatment, RAW264.7 cells were transfected with a miR-21-5p inhibitor. The levels of morphological damage and apoptosis of the colonic mucosal epithelial tissue were investigated using hematoxylin and eosin staining and a TUNEL staining assay, and then the colon macroscopic damage index and disease activity index were measured in rats. Western blot analysis was used to detect the protein expression levels of IL6R, STAT3, intracellular adhesion molecule 1 (ICAM-1), NF- $\kappa \mathrm{B}$, cleaved caspase-3, cleaved caspase-9 and Fas ligand (FasL). RT-qPCR detected the mRNA expression levels of miR-21-5p, IL6R, STAT3, ICAM-1, NF- $\kappa$ B, caspase-3, caspase-9 and FasL. An ELISA was performed to measure the levels of inflammatory cytokines. The viability and apoptosis levels of RAW264.7 cells were examined using MTT and flow cytometry assays. Additionally, STAT3 was investigated as a direct target
\end{abstract}

Correspondence to: Dr Xiaohong Lu, Department of Gastroenterology, Renmin Hospital of Wuhan University, Hubei Key Laboratory of Digestive System Disease, 238 Jiefang Road, Wuhan, Hubei 430060, P.R. China

E-mail: luxh1876@163.com

Key words: ulcerative colitis, microRNA-21-5p, signal transducer and activator of transcription 3, inflammatory, apoptosis of miR-21-5p in RAW264.7 cells using a dual-luciferase reporter assay. The results of the present study demonstrated that inflammation and apoptotic markers were revealed to be significantly downregulated following transfection with miR-21-5p inhibitors in RAW264.7 cells induced by LPS, and that cell viability was increased. Furthermore, STAT3 was confirmed to be a target of miR-21-5p in RAW264.7 cells. Collectively, these data demonstrated that miR-21-5p inhibition mediated the IL-6/STAT3 pathway in UC rats to decrease the levels of inflammation and apoptosis in RAW264.7 cells, and suggested that miR-21-5p may be an important therapy target in human UC.

\section{Introduction}

Inflammatory bowel disease (IBD) is a chronic non-specific intestinal inflammatory disease, like ulcerative colitis (UC) or Crohn's disease. Over the previous 2 decades, the morbidity and prevalence of UC has increased, becoming one of the modern incurable diseases, as determined by the World Health Organization (1). The duration, extent and severity of colonic mucosal inflammation is largely associated with the risk of cancer formation and promotion in UC (2). In order to cure $\mathrm{UC}$, early detection and studies examining its pathogenesis is crucial.

MicroRNAs (miRNAs) are small non-coding RNAs with the length of 19-24 nucleotides that bind with the 3'-untranslated regions (3'-UTR) of mRNA sequence to regulate the expression of its target genes $(3,4)$. At present, a number of studies have demonstrated that various miRNAs are closely associated with the process of $\mathrm{UC}$, indicating that miRNAs may be a therapeutic target for UC (5-9).

miR-21 has been suggested to serve a significant role in the development of IBD. A previous study demonstrated that the degree of intestinal inflammatory response, apoptosis of intestinal epithelial cells and their pathological scores were significantly decreased following the knockdown of miR-21 in Wistar rats treated with dextran sulfate sodium (DSS), as compared with the control group, which indicated that miR-21 may promote the occurrence and development of intestinal inflammation (10). Similarly, increased levels of miR-21 were identified in patients with UC, as compared with the control patients (11). However, the understanding of the regulation, role and targets of miR-21-5p remains limited. 
Several studies have described the association between miR-21-5p and signal transducer and activator of transcription 3 (STAT3): Gryshkova et al (12) identified an association among miR-21-5p, STAT3 and inflammatory responses in cardiac injury. In patients with celiac disease, miR-21-5p upregulation may have been caused by its target STAT3, indicating an increased activation of miR-21-5p in patients with Marsh 3C stage disease (13). An additional study demonstrated that STAT3 was upregulated in patients with UC and that the STAT3 expression increased with the severity of UC, suggesting that STAT3 may be an evaluation index of UC severity and prognosis and a new target in UC therapy (14). In addition, the expression levels of interleukin (IL)- 6 and IL6 receptor (IL6R) in UC rats were significantly increased, as compared with the control group (15). Wang et al (16) demonstrated that dandelion polysaccharides decreased the expression of IL-6 in UC rats and the protein expression of IL6R $\alpha$ and gp130 in the IL6R/STAT3 pathway, which decreased the transcriptional levels of STAT3 and IL6R mRNA and alleviated the inflammatory state in the colonic tissues of rats. Therefore, the IL6R/STAT3 pathway is associated with the process of UC, but the mechanism in which miR-21-5p mediates UC through the IL6R/STAT3 pathway remains to be elucidated.

In the present study, the role of miR-21-5p in UC was explored, with a particular focus on the effect of miR-21-5p on the IL6R/STAT3 signal pathway in UC and the regulation of inflammatory pathways and apoptosis-associated proteins in RAW264.7 cells.

\section{Materials and methods}

Human sera specimens. The study was approved by the Human Ethics Committee Review Board of Renmin Hospital of Wuhan University (Wuhan, China), and informed consent was obtained from each patient. Sera specimens were obtained from 45 patients with UC and 45 healthy individuals in the Renmin Hospital of Wuhan University (Wuhan, China) between May 2017 and June 2018. None of the patients had received prior treatment. All patients recruited for the present study were diagnosed with UC. The sera specimens were stored at $-80^{\circ} \mathrm{C}$ until further use. The study did not use patient names, initials, hospital numbers, or in any manner give information by which the individuals can be identified.

UC rat model. A total of 60 male Wistar rats (specific pathogen-free grade, 6 weeks, weighing 180-220 g) were obtained from Shanghai JiesiJie Experimental Animal Co., Ltd. Prior to the experiments, rats were maintained in an environmentally controlled room $\left(22^{\circ} \mathrm{C} \pm 2^{\circ} \mathrm{C}, 12: 12 \mathrm{~h}\right.$ light:dark cycle) with ad libitum access to food and water for 7 days, in order to acclimate to their new environment prior to initiation of the experiment. Animal experiments were approved and supervised by the Animal Care and Use and the Animal Ethics Committees at Renmin Hospital of Wuhan University. All rats were randomly divided into two groups $(n=30$ per group); the control and model groups. Following fasting for $4 \mathrm{~h}$, the rats in the model group were given ad libitum access to $5 \%$ DSS solution for 7 days, while the rats in the control group were given ad libitum access to purified water for 7 days. During the experiment, all rats received ad libitum access to food. At the time of sacrifice, the average weight of the control rats was $263 \mathrm{~g}$ and the average weight of the model rats was $180 \mathrm{~g}$. The rats received intraperitoneal anesthesia with $350 \mathrm{mg} / \mathrm{kg} 7 \%$ chloral hydrate prior to cervical dislocation. The rats exhibited no sign of peritonitis during the animal model.

Evaluation of disease activity index (DAI). From the beginning of the experiment, the general condition of rats was observed and recorded daily. The rat body mass was measured and the fecal characteristics and hematochezia were observed regularly every day. The DAI was assessed according to DAI score $(17,18)$.

Evaluation of colon macroscopic damage index (CMDI). All rats were anesthetized and sacrificed by cervical dislocation. The distal colon tissues ( $\sim 8 \mathrm{~cm}$ from the anus) at the same horizontal segment were used for the preparation of pathological tissue sections. The changes in the mucosa and serosal surfaces of the colon tissue were observed with the naked eye. The CMDI of colonic tissue was assessed according to the criteria for the assessment of microscopic colonic damage (19).

Evaluation of tissue damage index (TDI). A piece of fresh colonic tissue $(1.0 \times 1.0 \times 0.2 \mathrm{~cm})$ was collected and fixed in $4 \%$ paraformaldehyde at $4^{\circ} \mathrm{C}$ for $24 \mathrm{~h}$ in an embedding box, and then embedded in paraffin. Paraffin sections (3-4 $\mu \mathrm{m})$ were dewaxed with xylene for 10 min, dehydrated in 75 and $85 \%$ alcohol for $10 \mathrm{~min}$ and stained with hematoxylin for 2-3 min and $0.5 \%$ eosin for $1 \mathrm{~min}$ at room temperature. HE-stained samples were observed using a TS100 inverted optical microscope (CFI60 optical system; Nikon Corporation) at the magnification of 100. TDI of the colonic tissue was assessed according to the histological grading of colitis (20).

Cell transfection. RAW264.7 cells were purchased from American Type Culture Collection. RAW 264.7 cells $\left(1 \times 10^{5} / \mathrm{ml}\right)$ were seeded in 96 -well plates at a density of $1 \times 10^{5} / \mathrm{ml}$. RAW264.7 cells were induced by $1 \mathrm{mg} / 1$ LPS (Sigma-Aldrich; Merck KGaA) at room temperature for $24 \mathrm{~h}$. The miR-21-5p inhibitor and inhibitor control from Shanghai GenePharma Co., Ltd. were transfected into LPS-induced RAW264.7 cells using Lipofectamine ${ }^{\circledR} 2000$ reagent (Invitrogen; Thermo Fisher Scientific, Inc.) at $37^{\circ} \mathrm{C}$ with $5 \%$ $\mathrm{CO}_{2}$ for $48 \mathrm{~h}$. miR-21-5p inhibitor was 5'-UCAACAUCAGUC UGAUAAG and inhibitor control was 5'-CAGUACUUU UGUGUAGUAG. After $24 \mathrm{~h}$, the transfected RAW264.7 cells were used for subsequent experiments. The RAW264.7 cells were divided into three groups: Untransfected (control); cells transfected with inhibitor control (NC), and cells transfected with miR-21-5p inhibitor. The success of the transfection was examined by reverse transcription-quantitative polymerase chain reaction (RT-qPCR).

Dual-luciferase reporter assay. TargetScan software 7.2 (http://www.targetscan.org) identified that STAT3 was a potential target of miR-21-5p and a luciferase activity assay was performed to verify this prediction (21). The RAW264.7 cells were co-transfected with STAT3 3'UTR pmirGLO 
plasmid [containing wild-type (WT) STAT3 3'-UTR or mutant (MUT) STAT3 3'-UTR; Promega Corporation] and miR-21-5p inhibitor or NC vector using Lipofectamine ${ }^{\circledR} 2000$ reagent. After $24 \mathrm{~h}$, the fluorescence activity was measured by the Renilla-Lumi ${ }^{\mathrm{TM}}$ Plus Luciferase Assay kit (Beyotime Institute of Biotechnology) with the Dual-Luciferase Reporter System (Promega Corporation).The method of normalization was basing on the comparison with Renilla luciferase activity.

ELISA. The level of IL- 6 and tumor necrosis factor- $\alpha$ (TNF- $\alpha$ ) in rat sera and RAW264.7 cells was respectively determined by rat IL-6 ELISA kit (cat. no. SBJ-R0064) and rat TNF- $\alpha$ ELISA kit (cat. no. SBJ-R0040) (both, Nanjing SenBeiJia Biological Technology Co., Ltd.) according to the manufacturer's protocol.

Terminal deoxynucleotidyl-transferase-mediated dUTP nick end labeling (TUNEL) staining. The paraffin sections were dewaxed with xylene twice for $10 \mathrm{~min}$ and then cleaned with absolute alcohol for $5 \mathrm{~min}, 90 \%$ alcohol for $2 \mathrm{~min}$ and $70 \%$ alcohol for $2 \mathrm{~min}$, followed by the washing of distilled water for $2 \mathrm{~min}$. Then, tissue slides were treated with $20 \mu \mathrm{g} / \mathrm{ml}$ proteinase $\mathrm{K}$ and incubated at $20-37^{\circ} \mathrm{C}$ for $15-30 \mathrm{~min}$, followed by incubation with $50 \mu 1$ TUNEL solution at $37^{\circ} \mathrm{C}$ for $60 \mathrm{~min}$ in the dark. Finally, a drop of antifade mounting medium (Beyotime Institute of Biotechnology) was added for 5 min and samples observed by fluorescence microscopy (Invitrogen; Thermo Fisher Scientific, Inc.) at an excitation wavelength of 450-500 $\mathrm{nm}$ and an emission wavelength of 515-565 nm. Five fields of view were observed by fluorescent microscope.

MTT assay. Followed by transfection for $48 \mathrm{~h}$, cell viability was determined using Cell Proliferation and Cytotoxicity Assay kit (Beyotime Institute of Biotechnology) according to the manufacturer's protocol. The optical density value was measured at $490 \mathrm{~nm}$ with a Synergy ${ }^{\mathrm{TM}} 2$ Multi-function Microplate Reader (BioTek Instruments, Inc.).

Flow cytometry. Following transfection for $48 \mathrm{~h}$, an Annexin V-fluorescein isothiocyanate (FITC)/propidium iodide (PI) Apoptosis Detection kit (BD Biosciences) was used to analyze the apoptotic level of RAW264.7 cells. The RAW264.7 cells were collected with trypsin and washed with PBS, and then resuspended in $500 \mu 1$ HEPES buffer solution (Sigma-Aldrich; Merck KGaA) and incubated with $5 \mu \mathrm{l}$ Annexin V-FITC and $5 \mu \mathrm{PI}$ at room temperature for $15 \mathrm{~min}$ in the dark. Finally, a FACSVerse $^{\mathrm{TM}}$ flow cytometer with the FACSCanto II FACP Array ${ }^{\mathrm{TM}}$ software (v.3.0; BD Biosciences) was used to analyze the RAW264.7 cell apoptosis data.

Western blot analysis. Total proteins from colonic tissues and RAW264.7 cells were lysed using a radioimmunoprecipitation assay lysis buffer (Cell Signaling Technology, Inc.). A BCA Protein Assay kit (Pierce; Thermo Fisher Scientific, Inc.) was used to determine the protein concentration. Equal amounts of protein (30 $\mu \mathrm{g} /$ lane) were loaded on a $12 \%$ SDS-PAGE gradient gel to be separated and transferred to polyvinylidene fluoride membranes (EMD Millipore). Following blocking with $5 \%$ non-fat milk at $4^{\circ} \mathrm{C}$ for $2 \mathrm{~h}$, membranes were incubated overnight at $4^{\circ} \mathrm{C}$ with the following primary antibodies:
Anti-IL6R (1:500, cat. no., ab128008); anti-STAT3 (1:1,000; cat. no., ab68153); anti-intracellular adhesion molecule 1 (ICAM-1; 1:1,000; cat. no., ab171123); anti-NF-кB (1:1,000; cat. no., ab32360); anti-caspase-3 (1:500; cat. no., ab4051); anti-cleaved caspase-3 (1:500; cat. no., ab2302); anti-caspase-9 (1:1,000; cat. no., ab52298); anti-cleaved caspase-9 (1:1,000; cat. no., ab2324); and anti-Fas ligand (FasL; 1:1,000; cat. no., ab231011). All antibodies were purchased from Abcam, and then incubated with a horseradish peroxidase-conjugated secondary antibody (1:5,000; cat. no. sc-2357; Santa Cruz Biotechnology, Inc.). The membranes were subjected to enhanced chemiluminescence assay (Pierce; Thermo Fisher Scientific, Inc.) and detected using a ChemiDoc ${ }^{\mathrm{TM}}$ XRS+ imaging system (Bio-Rad Laboratories, Inc.). The blot densities were analyzed using the Media Cybernetics Gel-Pro Analyzer software 6.3 (Media Cybernetics, Inc.). GAPDH was used as the internal control.

$R T-q P C R$. Total RNA was extracted from colonic tissues or RAW264.7 cells using TRIzol ${ }^{\circledR}$ reagent (Thermo Fisher Scientific, Inc.). A total of $2 \mu \mathrm{g}$ RNA was used for the generation of cDNA using the High Capacity cDNA Reverse Transcription kit (Thermo Fisher Scientific, Inc.). RT-qPCR was performed by a TaqMan ${ }^{\mathrm{TM}}$ MicroRNA Assay kit (Applied Biosystems; Thermo Fisher Scientific, Inc.) for miR-21-5p and a Power SYBR Green qPCR Master Mix (Takara Biotechnology Co., Ltd.) for IL6R, STAT3, ICAM-1, $\mathrm{NF}-\kappa \mathrm{B}$, caspase -3 , caspase -9 and FasL, ccording to the manufacturer's protocols. The thermocycling conditions are as follows: Initial denaturation at $95^{\circ} \mathrm{C}$ for $2 \mathrm{~min} ; 38$ of cycles of denaturation at $95^{\circ} \mathrm{C}$ for $1 \mathrm{~min}$, annealing at $37^{\circ} \mathrm{C}$ for 1 min and elongation at $72^{\circ} \mathrm{C}$ for $2 \mathrm{~min}$; and a final extension at $72^{\circ} \mathrm{C}$ for $10 \mathrm{~min}$. The primer sequences for qPCR were as follows: U6 forward, 5'-CTCGCTTCGGCAGCACA-3' and reverse, 5'-AACGCTTCACGAATTTGCGT-3'; miR-21-5p forward, 5'-TAGCTTATCAGACTGATG-3' and reverse, 5'-CAGTGCGTGTCGTGGAGT-3'; GAPDH forward, 5'-GGAGTCCACTGGTGTCTTCA-3' and reverse, 5'-GGG AACTGAGCAATTGGTGG-3'; IL6R forward, 5'-CTCCTG CCAGTTAGCAGTCC-3' and reverse, 5'-TCTTGCCAGGTG ACACTGAG-3'; STAT3 forward, 5'-TGTCCGTCGTGGATC TGAC-3' and reverse, 5'-CCTGCTTCACCACCTTCTTG-3'; ICAM-1 forward, 5'-CCCTTCCCCCCAAAACTG-3' and reverse, 5'-GTCATTGTGAACACTGGCAGAAA-3'; NF-кB forward, 5'-CTGAACCAGGGCATACCTGT-3' and reverse, 5'-GAGAAGTCCATGTCCGCAAT-3'; caspase-3 forward, 5'-AAGGCAGAGCCATGGACCAC-3' and reverse, 5'-CTG GCAGCATCATCCACACAT-3'; caspase-9 forward, 5'-ATA CACCCTGGACTCGGATCC-3' and reverse, 5'-TGCTGA AGCTTCTCACAGTCC-3'; FasL forward, 5'-GACCCAGAA TACCAAGTGCAGA and reverse, 5'-CTGTTTCAGGAT TTAAGGTTGGA-3'. miR-21-5p and mRNA expression data were calculated relative to U6 and GAPDH, respectively, using the $2^{-\Delta \Delta C q}$ method (22).

Statistical analysis. All experimental data are presented as mean \pm standard deviation, and were analyzed with SPSS 19.0 (IBM Corp.). Comparisons between two groups were evaluated using s Student's t-test, and multiple comparisons between different groups were evaluated using a one-way 
A

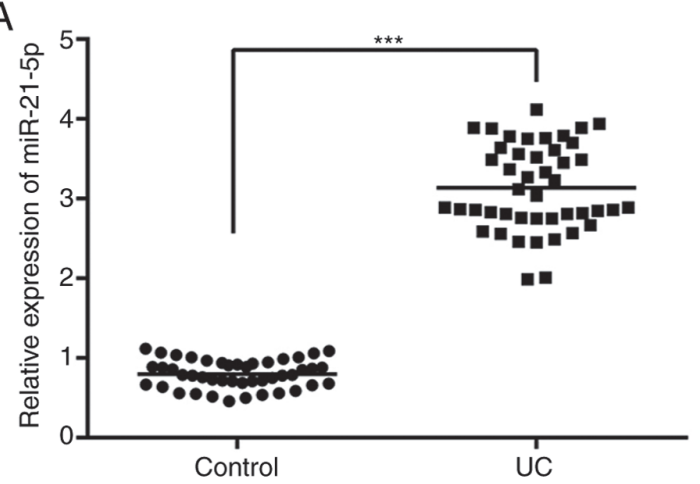

B

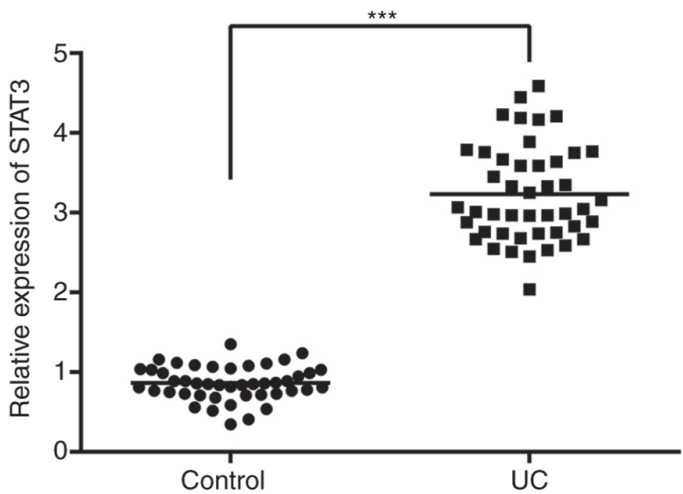

Figure 1. Expression of miR-21-5p and STAT3 in different groups, as detected by a reverse transcription-quantitative polymerase chain reaction assay. (A) miR-21-5p expression was increased in the UC group. (B) STAT3 expression was increased in the UC group. ${ }^{* * *} \mathrm{P}<0.001$ vs. control group. miR, microRNA; STAT3, signal transducer and activator of transcription 3; UC, ulcerative colitis.

A

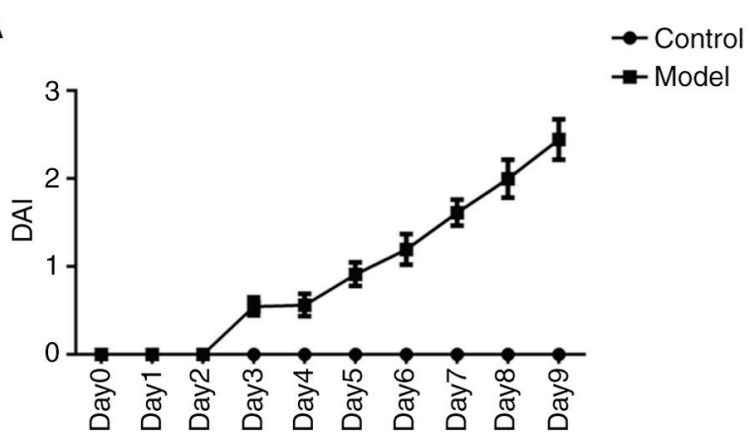

C
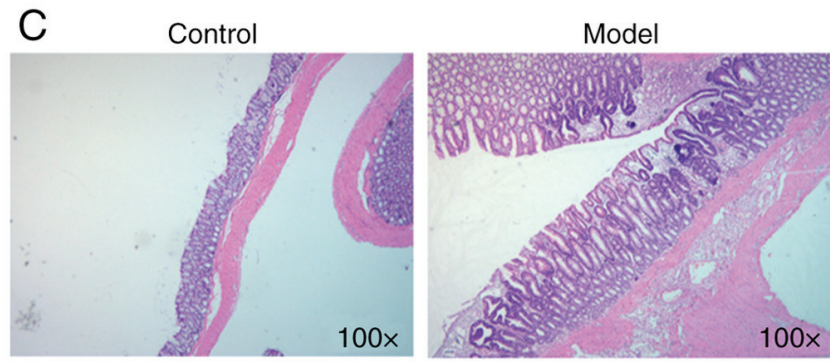
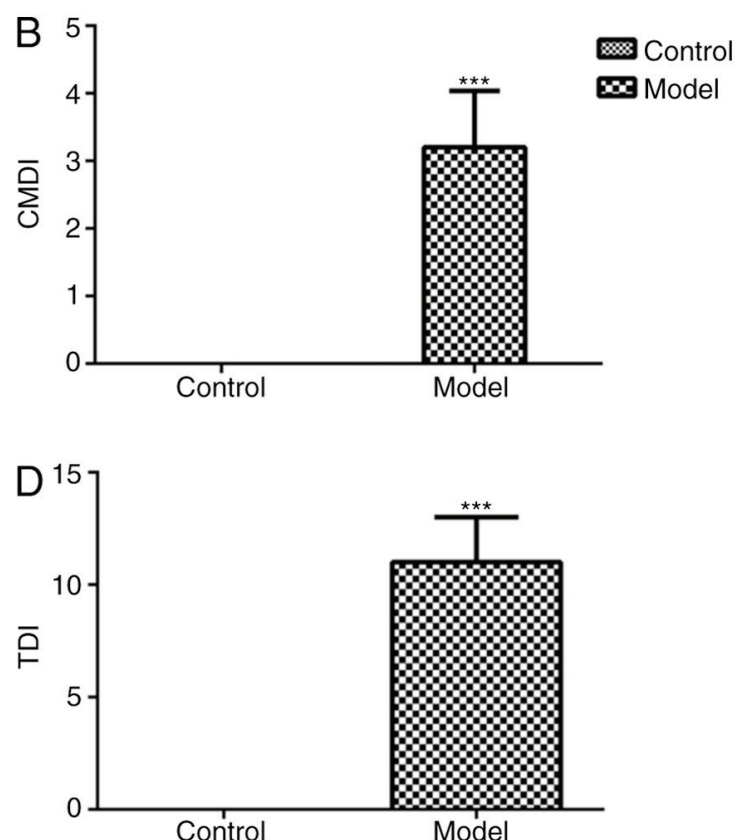

Figure 2. General condition and histological analysis. (A) DAI was increased in the UC model group. (B) CMDI was increased in the UC model group. (C) Representative images of colonic mucosal morphologies in each group. (D) TDI was increased in the UC model group. ${ }^{* * *} \mathrm{P}<0.001$ vs. control group. DAI, disease activity index; CMDI, colon macroscopic damage index; TDI, tissue damage index; UC, ulcerative colitis.

analysis of variance with Duncan's multiple-range test. $\mathrm{P}<0.05$ was considered to indicate a statistically significant difference.

\section{Results}

Expression of miR-21-5p and STAT3 in human sera. As indicated in Fig. 1A, the median of miR-21-5p in the control group was 0.78 and the median of miR-21-5p in the patients with UC was 3.12. As demonstrated in Fig. 1B, the median STAT3 level in the control group was 0.86 and the median STAT3 level in the patients with UC was 3.25 .

General condition and histological analysis. Compared with the control group, DAI increased following prolonged DSS administration (Fig. 2A). CMDI in the model group was markedly increased compared with that in the control group
(Fig. 2B). The colonic mucosal morphologies in each group were observed using a microscope. Clear layers of colonic structures, aligned mucosal epithelial cells, regular intestinal glands and no hyperemia and edema in the stroma were observed in the control group, while colonic mucosa edema, congestion and inflammatory infiltration were observed in the model group (Fig. 2C). This coincided with an increased damage score in the model group, as compared with the control group (Fig. 2D).

Expression of miR-21-5p in rat colon tissues. As demonstrated in Fig. 3A, the expression of miR-21-5p in colonic tissue of model group was increased compared with that in the control group.

Inflammation and apoptosis in rats with DSS-induced UC. As indicated in Fig. 3B and C, the concentrations of IL-6 
A

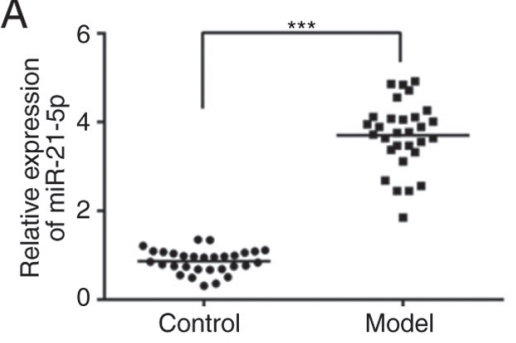

D
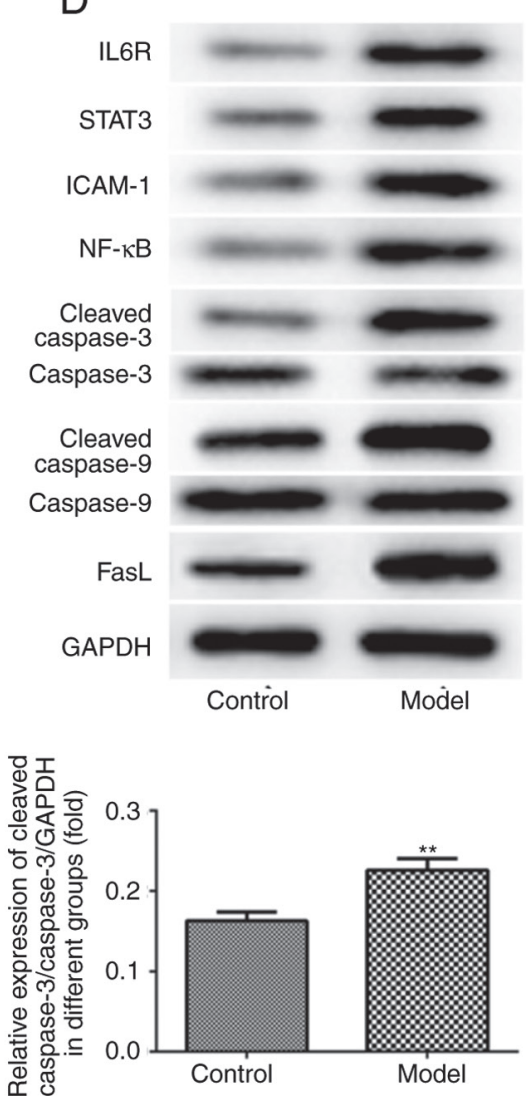

B
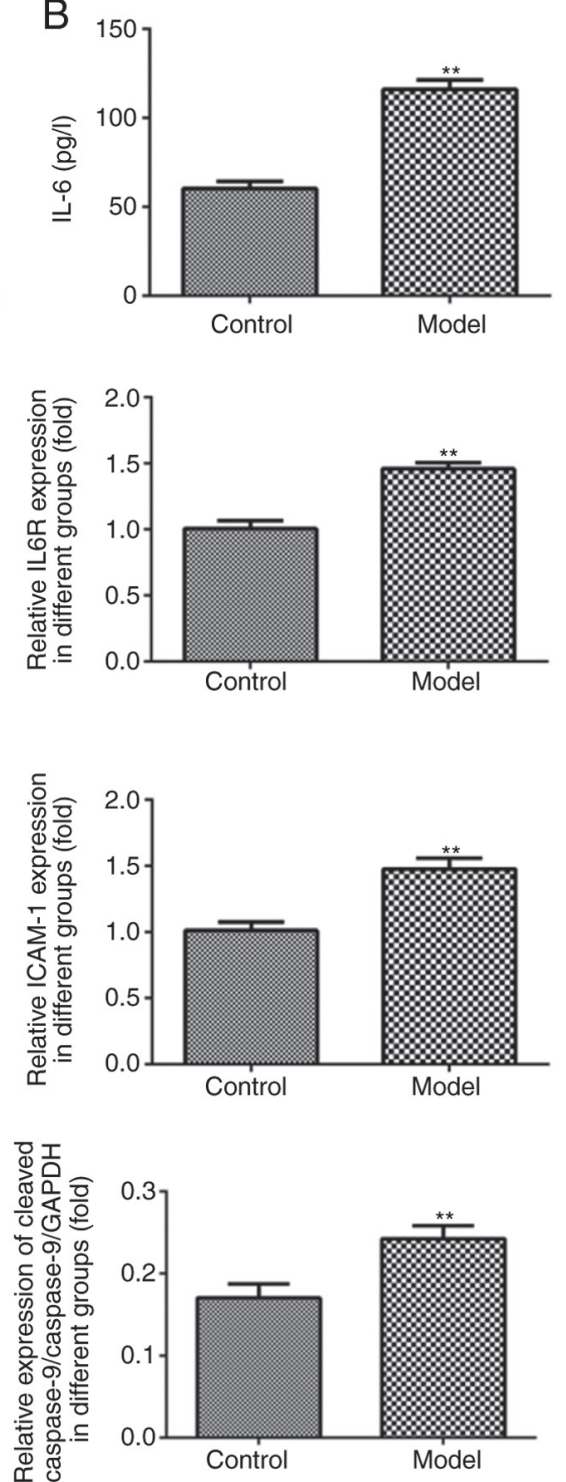
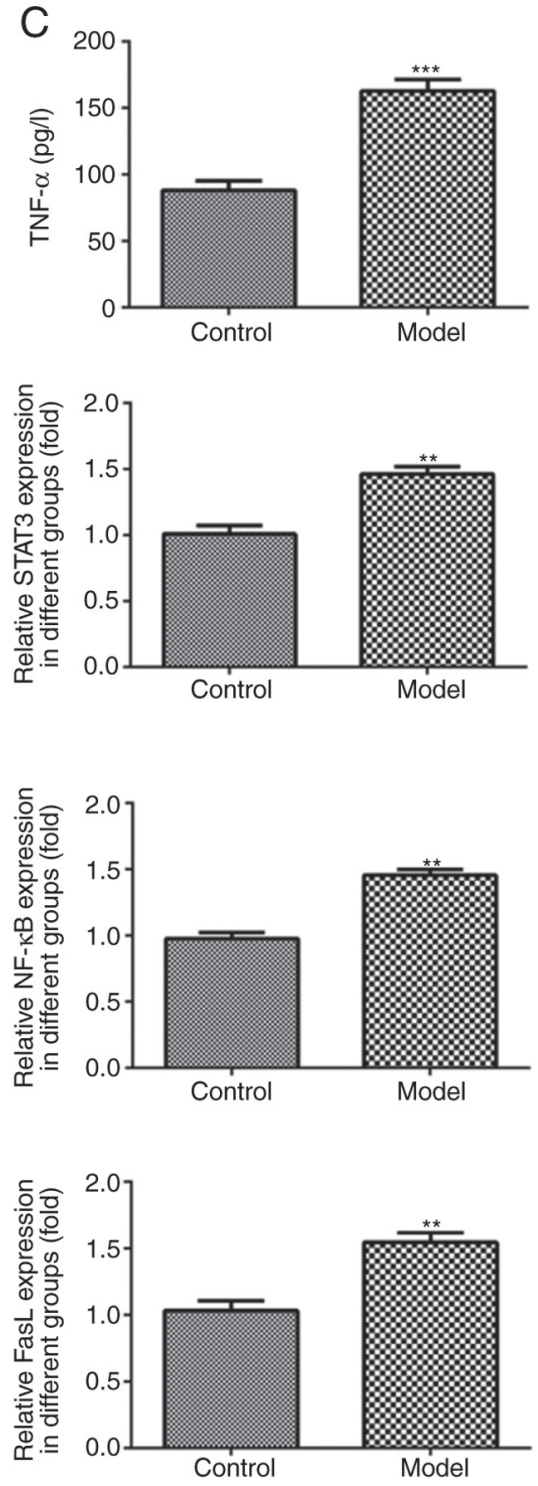

Figure 3. miR-21-5p expression, inflammation and apoptosis-associated protein expression in rat colon tissue. (A) miR-21-5p expression was increased in the UC model group.(B) The concentration of IL-6 was increased in the UC model group in rat sera. (C) The concentration of TNF- $\alpha$ was increased in the UC model group in rat sera. (D) The protein expression levels of IL6R, STAT3, ICAM-1, NF-кB, cleaved caspase-3, cleaved caspase-9 and FasL in rat colon tissue were examined using western blot analysis. ${ }^{* *} \mathrm{P}<0.01$ and ${ }^{* * *} \mathrm{P}<0.001$ vs. control group. miR, microRNA; UC, ulcerative colitis; IL-6, interleukin-6; TNF- $\alpha$, tumor necrosis factor- $\alpha$; IL6R, IL-6 receptor; STAT3, signal transducer and activator of transcription; ICAM-1, intracellular adhesion molecule-1; FasL, Fas ligand.

and $\mathrm{TNF}-\alpha$ in the rat sera were increased in the model group compared with those in the control group. Compared with the control group, the expression levels of IL6R, STAT3, ICAM-1, NF- $\kappa \mathrm{B}$, cleaved caspase-3, cleaved caspase-9 and FasL in the rat colon tissue were increased in the model group, as detected by western blot analysis (Fig. 3D). In addition, the level of apoptosis in the colonic epithelial cells was increased in the model group, as compared with that in the control group (Fig. 4).

miR-21-5p directly targets STAT3. Bioinformatics analysis was performed using the TargetScan tool to predict the potential targets of miR-21-5p, and the results indicated that STAT3 is one of the target genes of miR-21-5p (Fig. 5A). A dual-luciferase reporter assay was used to confirm this prediction. As indicated in Fig. 5B, the overexpression of
miR-21-5p clearly decreased the expression of STAT3 in the 3'UTR-WT group, while no difference was observed in the 3'UTR-MUT group, suggesting that miR-21-5p directly targets STAT3.

Effect of miR-21-5p on LPS-induced RAW264.7 cell inflammation, viability and apoptosis. The RAW264.7 cell line was used to investigate the role of miR-21-5p. As demonstrated in Fig. 6A, miR-21-5p was downregulated in RAW264.7 cells following transfection with miR-21-5p inhibitor, while no difference was observed between the $\mathrm{NC}$ and control groups. As indicated in Fig. 6B and C, the concentration levels of IL-6 and TNF- $\alpha$ in RAW264.7 cells were markedly decreased in the miR-21-5p inhibitor group, as compared with the NC and control groups. miR-21-5p inhibition suppressed the mRNA expression levels of IL6R, STAT3, ICAM-1, NF- $\kappa$ B, caspase-3, 

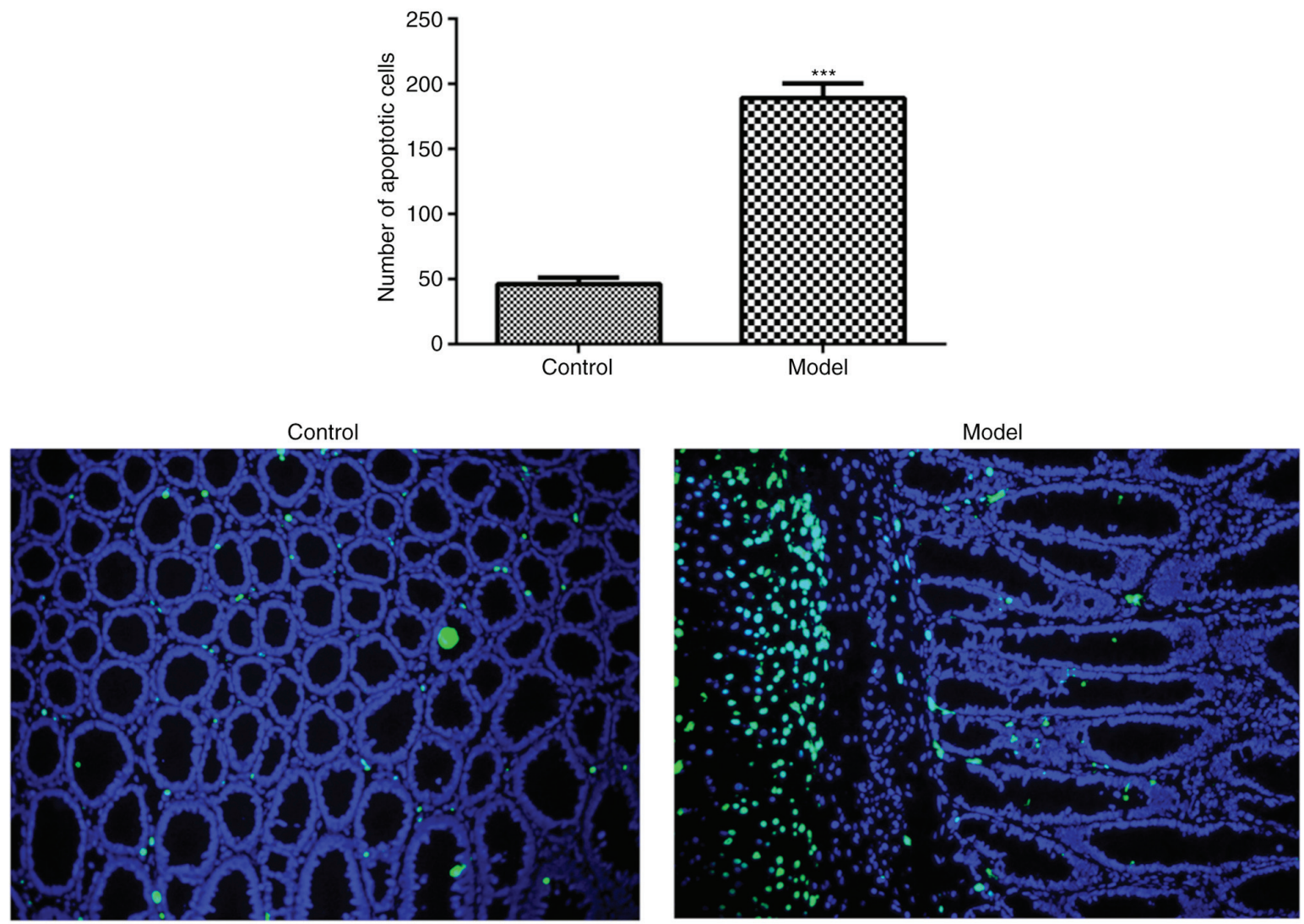

Figure 4. Apoptosis of colonic epithelial cells in the ulcerative colitis model and control groups (magnification, $\mathrm{x} 200$ ). ${ }^{* * *} \mathrm{P}<0.001$ vs. control group.

A STAT3 ACUCUUCAGUACAUAAUAAGCUU miR-21-5P AGUUGUAGUCAGAC-UAUUCGAU

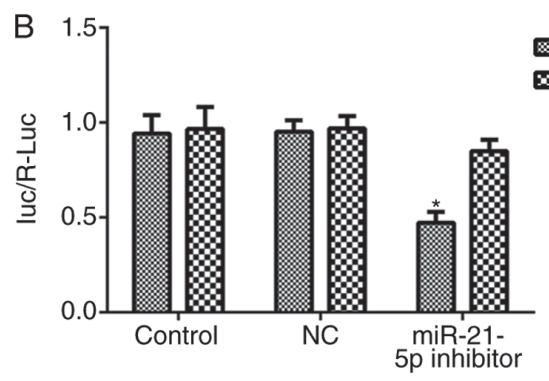

Figure 5. miR-21-5p directly targets STAT3. (A) Interaction between miR-21-5p and 3'-UTR of STAT3 was predicted by TargetScan. (B) Luciferase activity of a reporter containing a STAT3 3'-UTR-WT or 3'-UTR-MUT, which contained a mutation in the miR-21-5p binding site. ${ }^{*} \mathrm{P}<0.05$ vs. control group. ${ }^{~} \mathrm{P}<0.05$ vs. NC group. miR, microRNA; STAT3, signal transducer and activator of transcription; 3'-UTR, 3'-untranslated region; WT, wild type; MUT, mutated; NC, negative control.

caspase-9 and FasL in RAW264.7 cells, as compared with that in the $\mathrm{NC}$ and control groups (Fig. 6D). As shown in Fig. 7, the expression of IL6R, STAT3, ICAM-1, NF- $\kappa$ B, cleaved caspase-3, cleaved caspase-9 and FasL in RAW264.7 cells was decreased in RAW264.7 cells transfected with miR-21-5p inhibitor. MTT assay and flow cytometry analyses were conducted to investigate the effects of miR-21-5p on RAW264.7 cell viability and apoptosis. As demonstrated in Fig. 8, the viability of RAW264.7 cells was markedly increased while the apoptosis of RAW264.7 cells was obviously decreased in the miR-21-5p inhibitor group, as compared with the $\mathrm{NC}$ and control groups.

\section{Discussion}

In the present study, the expression levels of miR-21-5p and STAT3 was detected in the sera of patients with UC and tissues of UC rats. The general condition, histological changes, levels of inflammation-associated factors and apoptosis, and the mechanism and function of miR-21-5p in RAW264.7 cells, were also analyzed. It was identified that miR-21-5p inhibition mediated the IL6R/STAT3 pathway in UC rats to decrease the levels of inflammation and apoptosis, suggesting that miR-21-5p may be an important therapeutic target in human ulcerative colitis.

UC is a disease of the mucosal lining limited to the innermost layers of colon with cryptitis and crypt abscesses. Previous studies have demonstrated that miRNAs are associated with the progress of UCL: For example, miR-146 rs2910164 was identified to be associated with a decreased risk of UC in an Asian population (23). The miRNA-200b mediated inflammatory responses in an RAC-beta serine/threonine-protein kinase-dependent manner to suppress tumor development (24). miR-184 and miR-490-5p may regulate inflammatory signaling pathways, and attenuate inflammation and tissue injury in the colons of rats with DSS-induced UC (25). However, the understanding of the effect of miR-21-5p in DSS-induced UC remains limited.

In recent years, the role of miR-21-5p in a number of diseases has been studied due to its important functions (26-28). miR-21-5p downregulation may inhibit proliferation and apoptosis in esophageal squamous cell carcinoma (ESCC) cells and 

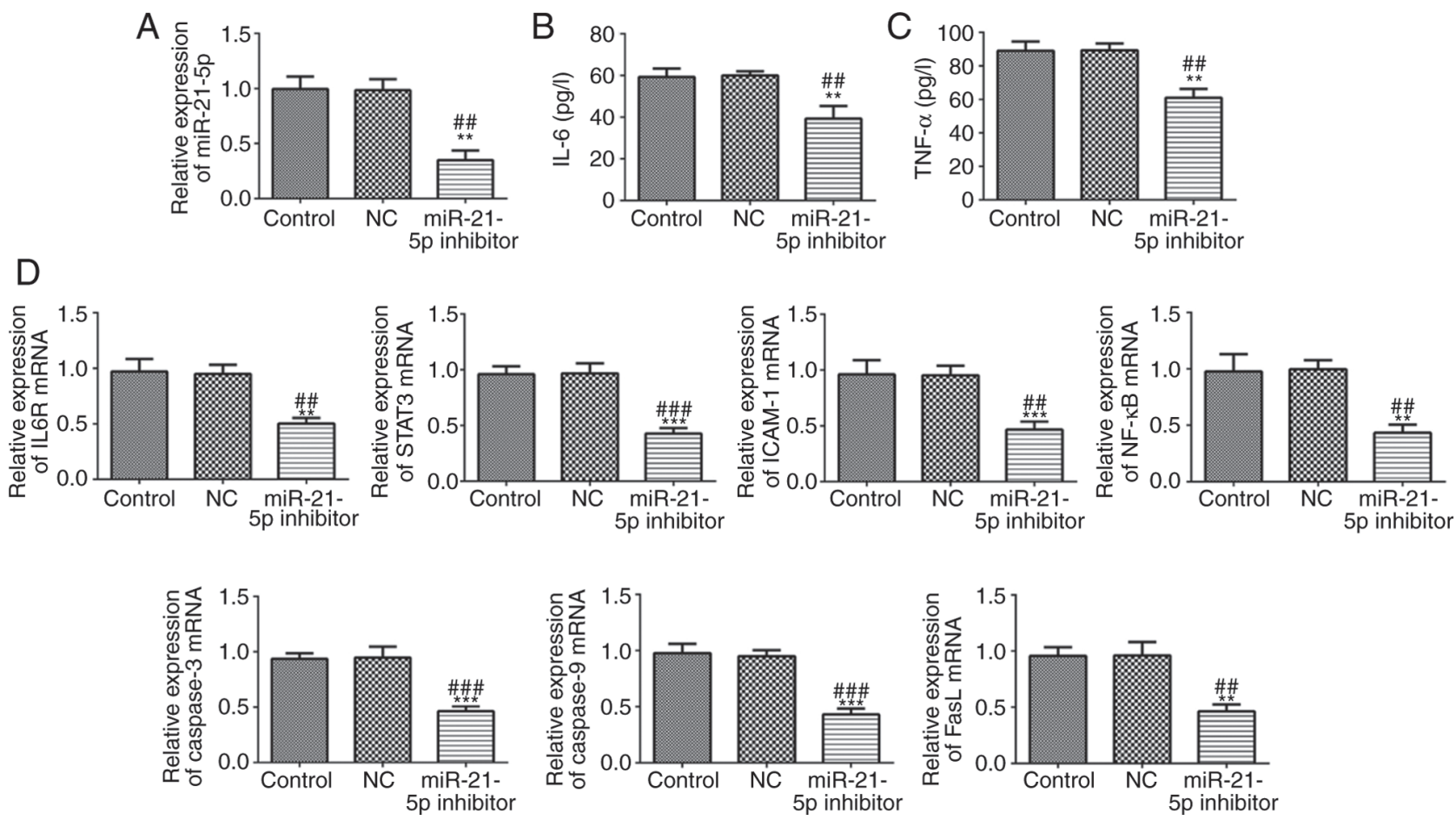

Figure 6. miR-21-5p inhibition improves the expression of inflammation and apoptosis-associated proteins. (A) miR-21-5p expression was decreased following cell transfection with miR-21-5p inhibitor. (B) The concentration of IL-6 was decreased in RAW264.7 cells transfected with miR-21-5p inhibitor. (C) The concentration of TNF- $\alpha$ was decreased in RAW264.7 cells transfected with miR-21-5p inhibitor. (D) The mRNA expression levels of IL6R, STAT3, ICAM-1, NF- $\mathrm{kB}$, caspase-3, caspase-9 and FasL were analyzed by reverse transcription-quantitative polymerase chain reaction. ${ }^{* * *} \mathrm{P}<0.01$ and ${ }^{* * * *} \mathrm{P}<0.001$ vs. control group. ${ }^{\# \#} \mathrm{P}<0.01$ and ${ }^{\# \# \#} \mathrm{P}<0.001$ vs. NC group. miR, microRNA; NC, negative control; IL-6, interleukin-6; tumor necrosis factor- $\alpha$; IL6R, IL-6 receptor; STAT3, signal transducer and activator of transcription; ICAM-1, intracellular adhesion molecule-1; FasL, Fas ligand.
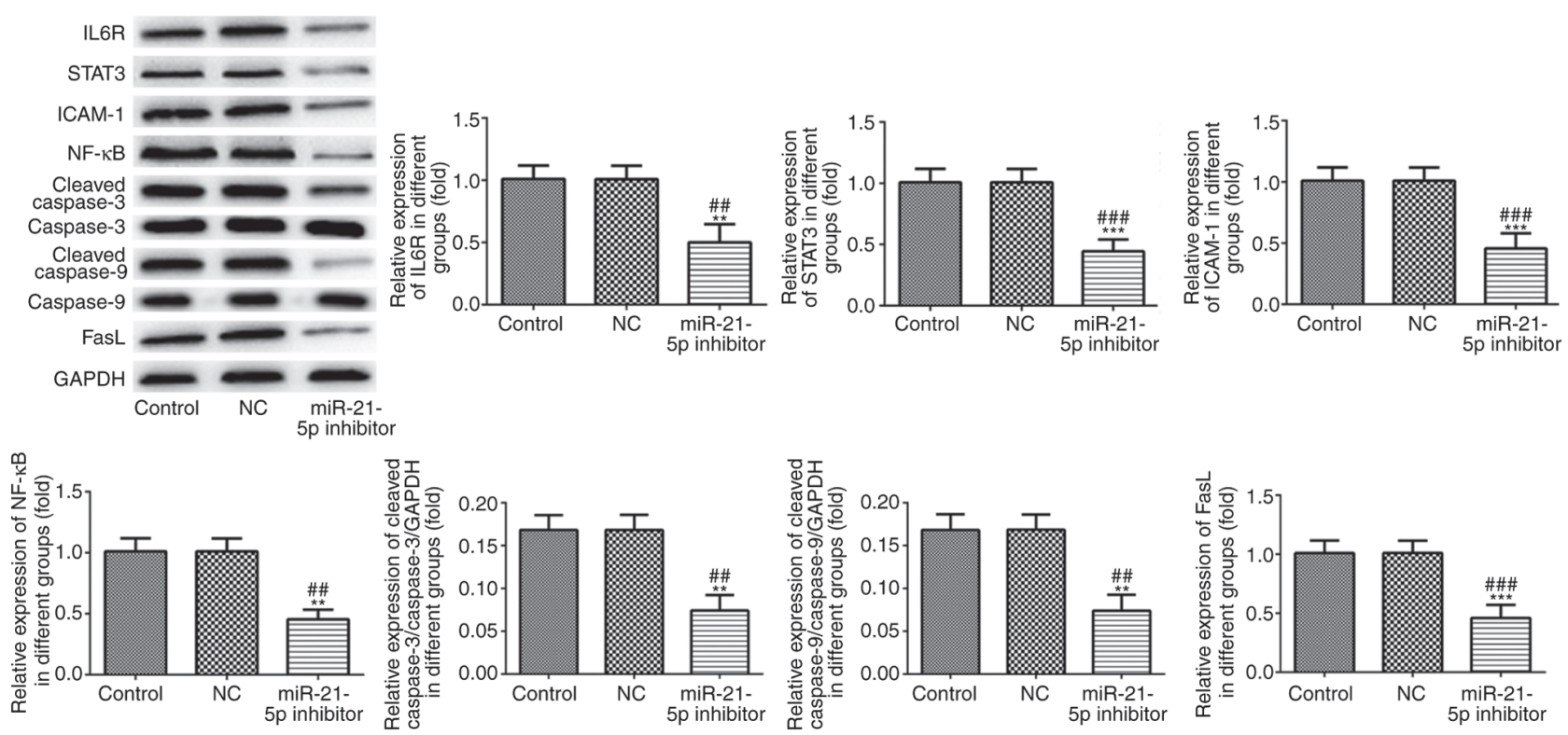

Figure 7. Protein expression levels of IL6R, STAT3, ICAM-1, NF-кB, cleaved caspase-3, cleaved caspase- 9 and FasL were analyzed by western blot analysis ${ }^{* *} \mathrm{P}<0.01$ and ${ }^{* * *} \mathrm{P}<0.001$ vs. control group. ${ }^{\# \#} \mathrm{P}<0.01$ and ${ }^{\# \# \#} \mathrm{P}<0.001$ vs. NC group. IL6R, IL-6 receptor; STAT3, signal transducer and activator of transcription; ICAM-1, intracellular adhesion molecule-1; FasL, Fas ligand; NC, negative control.

may represent a novel therapeutic target for the treatment of ESCC (29). miR-21-5p was overexpressed in response to the inflammatory cytokines in beta cell lines, and miR-21-5p inhibition could alleviate inflammation in beta cell lines (30). In traumatic brain injury, miR-21-5p suppressed inflammation by regulating the expression of inflammatory cytokines and NF-kB signaling and inhibited cellular apoptosis by regulating the expression of apoptosis factors and Akt signaling (31). We therefore hypothesized that miR-21-5p may be associated with inflammation and apoptosis in DSS-induced UC. In 
$\mathbf{A}$

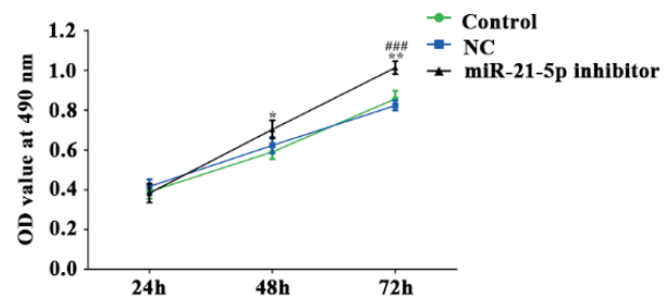

C

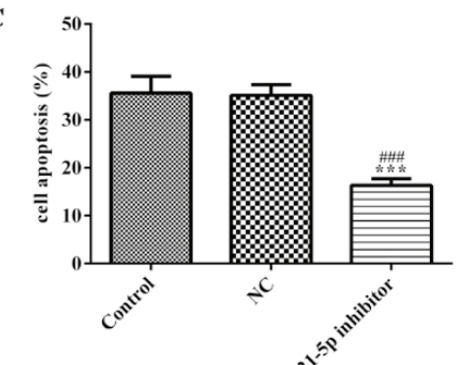

B

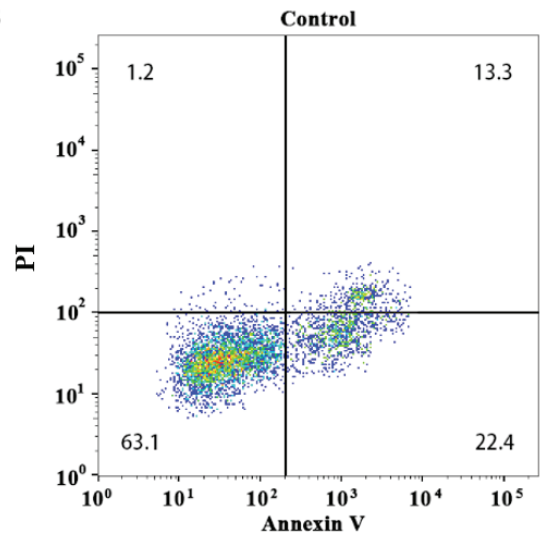

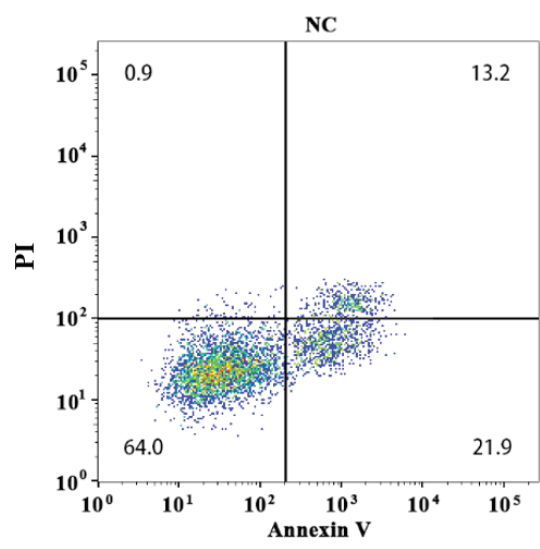

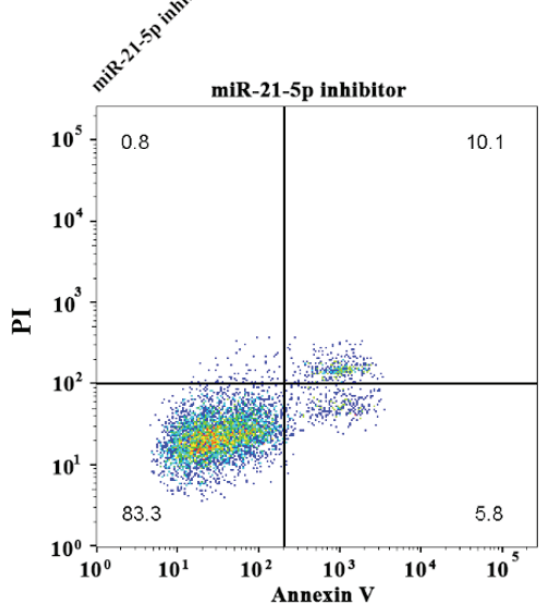

Figure 8. miR-21-5p inhibition promotes RAW264.7 cell viability and inhibits RAW264.7 cell apoptosis. (A) RAW264.7 cell viability was increased in RAW264.7 cells transfected with miR-21-5p inhibitor. vs. control group. ${ }^{\# \#} \mathrm{P}<0.001$ vs. NC group. (B) Cell apoptosis levels were evaluated by flow cytometry. (C) Apoptosis assay data were quantified. Apoptosis in the RAW264.7 cells was increased in RAW264.7 cells transfected with the miR-21-5p inhibitor. ${ }^{* *} \mathrm{P}<0.01$ and ${ }^{* * *} \mathrm{P}<0.001$ vs. control group. ${ }^{\# \# \#} \mathrm{P}<0.001$ vs. NC group. miR, microRNA; NC, negative control.

the present study, it was observed that miR-21-5p expression was clearly increased in the sera of patients with UC and rat colon tissue with DSS-induced UC. STAT3 is an inflammation-responsive transcription factor with a role in several types of cancer (32). STAT3 signaling is activated by inflammatory cytokines including IL-6, IL-10 and IL-22 (33). STAT3 and the STAT3 signaling pathway have been identified to serve a prominent role in colitis (34). Whether miR-21-5p regulates inflammation and apoptosis with STAT3 remains unknown. The results of the present study identified STAT3 to be a target of miR-21-5p, and demonstrated that miR-21-5p inhibition may regulate the expression of the STAT3-associatied pathway and apoptosis-associated proteins to alleviate inflammation and decrease the level apoptosis in RAW264.7 cells.

In conclusion, the present study indicated that miR-21-5p was downregulated in the sera and colon tissue of UC compared with healthy people and the control group. In addition, miR-21-5p inhibition may downregulate the expression of IL-6, TNF- $\alpha$, IL6R, STAT3, ICAM-1, NF- $\kappa$ B, cleaved caspase-3, cleaved caspase- 9 and FasL to alleviate the inflammation and apoptosis. In addition, miR-21-5p may exert its role partly by targeting STAT3, and miR-21-5p may be a therapeutic target for the treatment of UC.

\section{Acknowledgements}

Not applicable.

\section{Funding}

No funding was received.

\section{Availability of data and materials}

The datasets used and/or analyzed during the current study are available from the corresponding author on reasonable request.

\section{Authors' contributions}

XL conceptualized and developed the study design and performed the majority of the experiments. XL and YY acquired the data which analyzed by XL, YY and ST. XL and ST wrote the manuscript and XL and YY suggested appropriate modifications which were corrected by ST.

\section{Ethics approval and consent to participate}

The study was approved by the Human Ethics Committee Review Board of Renmin Hospital of Wuhan University, and informed consent was obtained from each patient.

\section{Patient consent for publication}

Informed consent was obtained from each patient.

\section{Competing interests}

The authors declare that they have no competing interests.

\section{References}

1. Ordás I, Eckmann L, Talamini M, Baumgart DC and Sandborn WJ: Ulcerative colitis. Lancet 380: 1606-1619, 2012. 
2. Gillen CD, Walmsley RS, Prior P, Andrews HA and Allan RN Ulcerative colitis and Crohn's disease: A comparison of the colorectal cancer risk in extensive colitis. Gut 35: 1590-1592, 1994.

3. Wang Z: MicroRNA: A matter of life or death. World J Biol Chem 1: 41-54, 2010

4. Kinose Y, Sawada K, Nakamura K and Kimura T: The role of MicroRNAs in ovarian cancer. Biomed Res Int 2014: 249393, 2014.

5. Shen N, Huang X and Li J: Upregulation of miR-129-5p affects laryngeal cancer cell proliferation, invasiveness, and migration by affecting STAT3 expression. Tumour Biol 37: 1789-1796, 2016.

6. Yang Y, Ma Y, Shi C, Chen H, Zhang H, Chen N, Zhang P, Wang F, Yang J, Yang J, et al: Overexpression of miR-21 in patients with ulcerative colitis impairs intestinal epithelial barrier function through targeting the Rho GTPase RhoB. Biochem Biophys Res Commun 434: 746-752, 2013.

7. Polytarchou C, Hommes DW, Palumbo T, Hatziapostolou M, Koutsioumpa M, Koukos G, van der Meulen-de Jong AE, Oikonomopoulos A, van Deen WK, Vorvis C, et al: MicroRNA214 is associated with progression of ulcerative colitis, and inhibition reduces development of colitis and colitis-associated cancer in mice. Gastroenterology 149: 981-992.e11, 2015

8. Ranjha R, Meena NK, Singh A, Ahuja V and Paul J: Association of miR-196a-2 and miR-499 variants with ulcerative colitis and their correlation with expression of respective miRNAs. PLoS One 12: e0173447, 2017.

9. Lewis A, Felice C, Kumagai T, Lai C, Singh K, Jeffery RR, Feakins R, Giannoulatou E, Armuzzi A, Jawad N, et al: The miR-200 family is increased in dysplastic lesions in ulcerative colitis patients. PLoS One 12: e0173664, 2017.

10. Shi CC: Study on the mechanism of miRNA-21 in regulating intestinal barrier function and inflammation related colon cancer. Shanghai Jiaotong University, 2014.

11. Thorlacius-Ussing G, Nielsen BS, Andersen V, Holmstrøm K and Pedersen AE: Expression and localization of miR-21 and miR-126 in mucosal tissue from patients with inflammatory bowel disease. Inflamm Bowel Dis 23: 739-752, 2017.

12. Gryshkova V, Fleming A, McGhan P, De Ron P, Fleurance R Valentin JP and Nogueira da Costa A: miR-21-5p as a potential biomarker of inflammatory infiltration in the heart upon acute drug-induced cardiac injury in rats. Toxicol Lett 286: 31-38, 2018.

13. Buoli Comani G, Panceri R, Dinelli M, Biondi A, Mancuso C, Meneveri R and Barisani D: miRNA-regulated gene expression differs in celiac disease patients according to the age of presentation. Genes Nutr 10: 482, 2015

14. Zhang T: Study on the relationship between intestinal barrier and STAT3 signaling pathway in ulcerative colitis. Tianjin Medical University, 2013 .

15. Li T, Zhu XD, Yang Y and Zhai YH: Effect of tongxie yaofang on the expression of IL- 6 and IL6R in the hypothalamus of rats with ulcerative colitis. Chin J Exp Trad Med Formulae 23: 103-108, 2017.

16. Wang Q, Bie YL, Wang D and Fan WT: Effect of dandelion polysaccharide on IL-6 /STAT3 signaling pathway in ulcerative colitis rats. Zhongguo Ying Yong Sheng Li Xue Za Zhi 33: 422-425, 2017 (In Chinese).

17. Murthy S, Cooper HS, Yoshitake H, Meyer C, Meyer CJ and Murthy NS: Combination therapy of pentoxifylline and TNFalpha monoclonal antibody in dextran sulphate-induced mouse colitis. Aliment Pharmacol Ther 13: 251-260, 1999.

18. Cooper HS, Murthy SN, Shah RS and Sedergran DJ: Clinicopathologic study of dextran sulfate sodium experimental murine colitis. Lab Invest 69: 238-249, 1993

19. Paiotti AP, Ribeiro DA, Silva RM, Marchi P, Oshima CT, Neto RA, Miszputen SJ and Franco M: Effect of COX-2 inhibitor lumiracoxib, the anti-TNF- $\alpha$ etanercept and your association on TNBS-induced colitis in Wistar rats. J Mol Histol 43: 307-317, 2012.
20. Dieleman LA, Palmen MJ, Akol H, Bloemena E, Peña AS, Meuwissen SG and Van Rees EP: Chronic experimental colitis induced by dextran sulphate sodium (DSS) is characterized by Th1 and Th2 cytokines. Clin Exp Immunol 114: 385-391, 1998.

21. Agarwal V, Bell GW, Nam J and Bartel DP: Predicting effective microRNA target sites in mammalian mRNAs. Elife 4: e05005, 2015.

22. Livak KJ and Schmittgen TD: Analysis of relative gene expression data using real-time quantitative PCR and the 2(-Delta Delta C(T)) method. Methods 25: 402-408, 2001

23. Li Z, Wang Y and Zhu Y: Association of miRNA-146a rs2910164 and miRNA-196 rs11614913 polymorphisms in patients with ulcerative colitis: A meta-analysis and review. Medicine (Baltimore) 97: e12294, 2018.

24. Deng S, Wang H, Fan H, Zhang L, Hu J, Tang Q, Shou Z, Liu X, Zuo D, Yang J, et al: Over-expressed miRNA-200b ameliorates ulcerative colitis-related colorectal cancer in mice through orchestrating epithelial-mesenchymal transition and inflammatory responses by channel of AKT2. Int Immunopharmacol 61: 346-354, 2018.

25. Huang Y, Ma Z, Cui YH, Dong HS, Zhao JM, Dou CZ, Liu HR, $\mathrm{Li} \mathrm{J}$ and Wu HG: Effects of herb-partitioned moxibustion on the miRNA expression profiles in colon from rats with DSS-induced ulcerative colitis. Evid Based Complement Alternat Med 2017: 767301, 2017

26. Liu Z, Yu M, Fei B, Fang X, Ma T and Wang D: miR-21-5p targets PDHA1 to regulate glycolysis and cancer progression in gastric cancer. Oncol Rep 40: 2955-2963, 2018.

27. Fromm B, Tosar JP, Lu Y, Halushka MK and Witwer KW: Human and cow have identical miR-21-5p and miR-30a-5p sequences, which are likely unsuited to study dietary uptake from cow milk. J Nutr 148: 1506-1507, 2018.

28. Li Q, Li B, Li Q, Wei S, He Z, Huang X, Wang L, Xia Y, Xu Z, $\mathrm{Li} \mathrm{Z}$, et al: Exosomal miR-21-5p derived from gastric cancer promotes peritoneal metastasis via mesothelial-to-mesenchymal transition. Cell Death Dis 9: 854, 2018.

29. Li XH, Chen D, Li M, Gao X, Shi G and Zhao H: The CADM2/Akt pathway is involved in the inhibitory effect of miR-21-5p downregulation on proliferation and apoptosis in esophageal squamous cell carcinoma cells. Chem Biol Interact 288: 76-82, 2018.

30. Lakhter AJ, Pratt RE, Moore RE, Doucette KK, Maier BF, DiMeglio LA and Sims EK: Beta cell extracellular vesicle miR-21-5p cargo is increased in response to inflammatory cytokines and serves as a biomarker of type 1 diabetes. Diabetologia 61: 1124-1134, 2018.

31. Ge XT, Huang S, Gao H, Han Z, Chen F, Zhang S, Wang Z, Kang C, Jiang R, Yue S, et al: miR-21-5p alleviates leakage of injured brain microvascular endothelial barrier in vitro through suppressing inflammation and apoptosis. Brain Res 1650: 31-40, 2016.

32. Hua Y, Drew P and Richard J: STATs in cancer inflammation and immunity: A leading role for STAT3. Nat Rev Cancer 9: 798-809, 2009.

33. Dang V, Barbi J, Yang HY, Jinasena D, Yu H, Zheng Y, Bordman Z, Fu J, Kim Y, Yen HR, et al: Control of T(H)17/T(reg) balance by hypoxia-inducible factor 1. Cell 146: 772-784, 2011.

34. Liu JZ, Suzanne VS, Hailiang H, Ng SC, Alberts R, Takahashi A, Ripke S, Lee JC, Jostins L, Shah T, et al: Association analyses identify 38 susceptibility loci for inflammatory bowel disease and highlight shared genetic risk across populations. Nat Genet 47: 979-986, 2015

This work is licensed under a Creative Commons Attribution-NonCommercial-NoDerivatives 4.0 International (CC BY-NC-ND 4.0) License. 